\title{
COMPARISON OF PERCEPTUAL AND PHYSIOLOGICAL VARIABLES OF RUNNING ON A TRACK, MOTORIZED TREADMILL, AND NON-MOTORIZED CURVED TREADMILL AT INCREASING VELOCITY
}

\author{
Veronika Myran Wee, Erna von Heimburg, \\ ROLAND VAN DEN TillaAR \\ Department of Sports Science and Physical Education, \\ Nord University, Levanger, Norway
}

\begin{abstract}
The aim of this study was to compare perceptual and physiological variables between running on three different modalities - an indoor athletics track, a motorized treadmill, and a non-motorized curved treadmill - for $1000 \mathrm{~m}$ at three different velocities. Ten male athletes (age $24 \pm 3$ years, body mass $69.8 \pm 6.91 \mathrm{~kg}$, height $1.80 \pm 0.06 \mathrm{~m}, \mathrm{VO}_{2 \text { peak }} 69.0 \pm 6.70 \mathrm{ml} / \mathrm{kg} /$ $\mathrm{min}$ ) conducted three $1000 \mathrm{~m}$ laps at increasing velocity on three different running modalities. The athletes had a 3-minute recovery between each lap, where the rate of perceived exertion (RPE) was registered and the blood lactate concentration and heart rate were measured. Oxygen uptake was measured using a portable metabolic analyser. The physiological (oxygen uptake, heart rate, and blood lactate concentration) and perceptual (RPE) variables were higher when running on a non-motorized curved treadmill compared with running on the track or a motorized treadmill. No differences were found between running on a motorized treadmill and the track except for the RPE, which was lower when running on the track compared with the motorized treadmill. Running on a non-motorized curved treadmill at three different velocities results in a higher oxygen uptake (37\%) and heart rate (22\%) and is subjectively much harder than running on a track or a motorized treadmill at the same velocities. The difference is around $4 \mathrm{~km} / \mathrm{h}$ when comparing the physiological and perceptual responses. Thus, when performing training sessions on a non-motorized curved treadmill, subjects should subtract $4 \mathrm{~km} / \mathrm{h}$ from their regular pace on a track or motorized treadmill to get
\end{abstract}


the same response considering oxygen uptake, heart rate, RPE and blood lactate concentration.

Keywords: rate of perceived exertion; blood lactate concentration; heart rate; oxygen uptake

\section{INTRODUCTION}

During running, the energy cost of the task performed depends upon a number of biomechanical, physiological, and environmental factors. Running modality is an environmental factor that can affect both biomechanical and physiological factors and can have a great impact on runners' energy costs during training or a race. Running on a track has generally been found to incur greater energy costs compared to running on a motorized treadmill [25]. This may be due to a number of factors, such as air resistance when running on a track, visual cues from moving surroundings, or the athletes' extent of familiarity with the chosen modality [12]. The effect of air resistance becomes more pronounced at high running speeds, and higher differences in energy costs between track running and motorized-treadmill running are therefore likely to be observed as velocity increases [4]. Other factors that can cause differences in perceptual and physiological responses between track and motorized-treadmill running are characteristics of the running surface, and thus the momentum runners gain from the moving treadmill belt or a change in locomotion characteristics on the various running surfaces [12]. Yet, motorized treadmills are widely used and considered valid for the measurement of overground running performance. Jones and Doust [12] emphasize the use of a $1 \%$ treadmill gradient to achieve the most strongly correlated oxygen uptake $\left(\mathrm{VO}_{2}\right)$ measures between such running modalities.

Although the relationship between energy costs on a motorized treadmill and a track has been investigated to a certain degree, fewer studies have been conducted on the energy costs on flat non-motorized treadmills. Nonmotorized treadmills, in contrast to motorized treadmills, do not have a motor to create belt motion but instead rely on the athlete to drive the belt. It is suggested that flat non-motorized treadmills have greater resemblance to track running compared to motorized treadmills. For instance, opposite to running on a motorized treadmill, the runner dictates the speed of the treadmill belt on the non-motorized flat treadmill with every step, consistent with track running, whereas on a motorized treadmill the runner follows 
the speed of the treadmill. Therefore, the flat non-motorized treadmill belt cannot serve as a motivator for the runner to maintain a high and consistent running speed during the performance of the task [23]. The flat non-motorized treadmill belt also forces the leg to actively push through on each step, which is the same as for track locomotion [6]. On the other hand, the power required to propel the treadmill belt on non-motorized treadmills increases with speed [13], due to high intrinsic resistance of the running belt [6]. This differs significantly from both track and motorized-treadmill running, along with the need to accelerate the treadmill belt between steps [10]. When it comes to energy costs on flat non-motorized treadmills, studies utilizing flat non-motorized treadmills have consistently shown higher energy costs in comparison with motorized treadmills, even at walking speeds [5, 9, 21]. Test subjects have also commented that the exercise was more difficult on flat non-motorized treadmills and that locomotion on non-motorized ergometers felt similar to running up an incline [14].

Non-motorized treadmills in various and improved variants have become readily available to sports scientists and the general public in the last few years. The Force, non-motorized treadmill (Woodway, Waukesha, WI, USA) works by the act of pushing backwards on a flat treadmill belt when wearing a harness, whereas a non-motorized curved treadmill (NMCT) (Woodway, Waukesha, WI, USA) works by actively pushing backwards on a curved treadmill belt. Both the Force non-motorized treadmill $[10,13]$ and the Woodway Curve $[8,16]$ have demonstrated good validity and reliability for sprint performance assessment in the laboratory, where motorized treadmills are not suitable due to restrictions in acceleration [23].

Only a few studies have investigated energy expenditure on a curved nonmotorized treadmill $[18,22,23]$. In the studies of McCarron et al. [18] and Stevens et al. [23], the subjects had to perform a $5-\mathrm{km}$ trial on an NMCT. Only in the study of Stevens et al. [23] energy expenditure was measured by oxygen uptake during the $5-\mathrm{km}$ trial on the NMCT. They compared running performance on the NMCT with running on an outdoor $400-\mathrm{m}$ athletic track. The total running time was $22 \%$ longer while running on the NMCT compared to running on the track. No difference was found in mean oxygen uptake, but blood lactate concentration and rate of perceived exertion (RPE) were significantly higher at the end of the non-motorized trial compared to the track trial, indicating that it was harder to run on an NMCT. However, Stevens et al. [23] used a time trial to compare two running modalities (track and non-motorized treadmill running), which caused the subjects to run at different velocities during the trails. This made it difficult to compare 
how much energy the subjects used when running at the same velocity on different running modalities.

Knowing the energy expenditure at the same running velocity on different running modalities would make it easier to compare the effects of these running modalities upon perceptual and physiological responses and performance. Because the characteristics of the treadmill belt on a non-motorized treadmill require higher power as the speed increases [13], investigating energy costs for graded exercise intensities is necessary. Only Smoliga et al. [22] have compared physiological and perceptual responses when walking and running on an NMCT and a motorized treadmill at the same velocities (walking: $1.34 \mathrm{~m} / \mathrm{s}$; running: $2.24 \mathrm{~m} / \mathrm{s}$ ), and they found that energy expenditure was higher when walking and running on an NMCT. However, in this study, only two velocities at a low pace $(4.8 \mathrm{~km} / \mathrm{h}$ and 8 $\mathrm{km} / \mathrm{h}$ ) were used, and they were not compared with running on a track. Furthermore, velocities that are regular in training for endurance-trained subjects were not used.

Therefore, the aim of the current study was to compare perceptual responses (RPE) and physiological responses (oxygen uptake, heart rate, and blood lactate concentration) when endurance trained subjects ran $1000 \mathrm{~m}$ intervals on an NMCT compared with running on an indoor track, and a motorized treadmill at three increasing intensities $(12 \mathrm{~km} / \mathrm{h}, 14 \mathrm{~km} / \mathrm{h}$, and $16 \mathrm{~km} / \mathrm{h}$ ). It was hypothesized that perceptual responses (RPE) and physiological responses (oxygen uptake, heart rate and blood lactate concentration) on an NMCT would be higher than on a motorized treadmill or track when running $1000 \mathrm{~m}$ laps due to the high intrinsic resistance of the running belt [23].

\section{MATERIALS AND METHODS}

To compare well-trained male athletes' perceptual and physiological responses to running on an indoor track, a motorized treadmill, and an NMCT, a within-subjects design was used. The independent variables were the type of modality (motorized treadmill, NMCT, or track), and the dependent variables were the perceptual (RPE) and physiological responses (oxygen uptake, heart rate, and blood lactate concentration).

\section{Subjects}

Ten well-trained male subjects participated in the present study. The group of subjects consisted of six long-distance runners, two middle-distance 
runners, one biathlete, and one cross-country skier (age $24 \pm 3.1$ yrs., body mass $69.8 \pm 6.9 \mathrm{~kg}$, body height $1.80 \pm 0.06 \mathrm{~m}, \mathrm{VO}_{2 \text { peak }} 69.0 \pm 6.9 \mathrm{ml} / \mathrm{kg} / \mathrm{min}$ ) who perform on the national level in their sport. The subjects were familiar with running on a motorized treadmill and on an indoor track. All subjects performed one running session on an NMCT at least one week prior to their actual test on this treadmill to familiarize themselves with this running modality. Subjects were informed of experimental procedures before signing a written consent form to participate, and they could withdraw from the study at any point. The study was conducted with the approval of the local ethics committee and in accordance with the principles outlines in the Declaration of Helsinki and current ethical standards in sports and exercise research.

\section{Procedures}

Subjects performed three tests each: the first on an indoor $200 \mathrm{~m}$ Mondo track (Mondo Spa, Gallo, Italy), the second on a motorized treadmill $(\mathrm{h} / \mathrm{p} /$ cosmos quasar ${ }^{\circ}$, Germany), and the third on an NMCT, Woodway Curve (Woodway, Waukesha, WI, USA). The order of the tests was not randomized, because the maximal $1000 \mathrm{~m}$ time had to be performed on the track, before it was possible to compare it with running on a motorized treadmill. The second run was on a motorized treadmill, since all subjects had a lot of experience with running on this modality and therefore did not need a familiarization session. After the runs on this modality they performed a familiarization session on the NMCT to avoid a learning effect on this modality. The motorized treadmill incline was set to $1 \%$ to mimic the overground running modality [12]. The tests were performed on separate days with at least 48 hours in between. In order to reduce the variability of testing, the time of day, the shoes worn, and the testing equipment were standardized for the three tests. Room temperature was centrally controlled and set to $18^{\circ} \mathrm{C}$.

Subjects were instructed not to perform vigorous activity for 24 hours prior to each test. Before the tests, the height (KaWe PERSON-CHECK ${ }^{-}$ height measuring device) and body weight (Soehnle Professional 7730) of each subject were measured. The subjects then put on a sports watch, a paired heart rate monitor, and a small backpack with a MetaMax II portable metabolic measurement system (MetaMax II Portable CPX Cortex Device, Leipzig, Germany) placed inside. The MetaMax II was connected to a facemask covering the subjects' nose and mouth. The backpack straps were adjusted and the mask fitted before starting the test. Then, the subjects 
performed a 5-min warm-up at a self-selected speed on the same running modality that the test was to be conducted on. The protocol involved running $1000 \mathrm{~m}$ laps at three pre-set running velocities $(12 \mathrm{~km} / \mathrm{h}, 14 \mathrm{~km} / \mathrm{h}$, and $16 \mathrm{~km} / \mathrm{h}$ ) and a maximal $1000 \mathrm{~m}$ lap with a 4-5-min recovery between each lap, which was enough time that the heart rate returned to baseline (heart rate before first $1000 \mathrm{~m}$ ). These velocities were chosen since they were regularly used in training on the track and were all to be expected to be lower than the anaerobic threshold (except the maximal $1000 \mathrm{~m}$ lap). The pre-set velocities on the track were guided by a control sound every $100 \mathrm{~m}$ during each $1000 \mathrm{~m}$. All subjects started with the protocol on the indoor track to establish the running velocity on the maximal $1000 \mathrm{~m}$ run. The average velocity from this maximal $1000 \mathrm{~m}$ run on the track was then used again as the velocity on the motorized and non-motorized treadmills to investigate if there were differences in maximal performance between the running modalities. Heart rate was continuously measured by a Garmin 910XT monitor (Garmin Ltd., Schaffhausen, Switzerland) and registered along with the RPE on a 6-20 scale, where 6 refers to no exertion at all and 20 is maximal exertion [2] immediately after finishing each $1000 \mathrm{~m}$ lap. A blood lactate concentration test was performed within the first minute of the 4-5-min recovery time between the laps. Blood samples $(0.5 \mu \mathrm{L}$ whole blood) were drawn from a fingertip for blood lactate analysis using a Lactate Pro (Arkray Lactate Pro, Shiga, Japan). The fingertip was dipped in sterile water and wiped with a piece of paper before getting a needle stick with a sterile lancet (Microlet 2 Lancing Device). The first blood drop was wiped off before collecting a blood sample with a test strip. The Lactate Pro was calibrated prior to each new test using a Lactate ProTM calibration strip. Several investigators have examined this analyzer and found it accurate and reliable $[17,19,24]$.

Oxygen uptake was determined and read from a MetaMax portable metabolic measurement system (MetaMax II, Leipzig, Germany). The MetaMax II measured oxygen uptake on a breath-by-breath basis and determined the volume of oxygen consumed per minute. Prior to the first test on each new test day, and thereafter for every second test, the MetaMax II was calibrated using a two-point calibration involving a calibration against ambient air and a commercial gas of known concentration of $\mathrm{O}_{2}(16 \%)$ and $\mathrm{CO}_{2}(4 \%)$. A volume calibration for the MetaMax II was performed between each new test using a 3-litre high-precision calibration syringe (Calibration syringe D; SensorMedics, Yorba Linda, CA, USA). Oxygen uptake and heart rate were measured continuously during the whole protocol. Heart rate, RPE, 
and blood lactate concentration were measured and registered immediately after three different submaximal stages of $1000 \mathrm{~m}$ at increasing intensities and maximal $1000 \mathrm{~m}$ run on each of the different running modalities. Oxygen uptake for each $1000 \mathrm{~m}$ run was calculated as the average oxygen uptake value from the last minute during that run on each modality, which was used for further analysis. To represent the subjects' $\mathrm{VO}_{2 \text { peak }}$ for this study, the highest average value observed during one of the $1000 \mathrm{~m}$ runs on one of the three running modalities was chosen for further analysis.

\section{Statistical analyses}

Differences in oxygen uptake, heart rate, blood lactate concentration, and RPE between trials were examined using a repeated 3 (modality: NMCT, motorized treadmill, indoor track) x 4 (velocity: $12 \mathrm{~km} / \mathrm{h}, 14 \mathrm{~km} / \mathrm{h}, 16 \mathrm{~km} / \mathrm{h}$, maximal) analysis of variance (ANOVA) design. Post hoc analysis with Holm-Bonferroni correction was conducted to locate differences. Statistical analysis was performed using SPSS (version 21.0; SPSS, Inc., Chicago, IL). The subjective rating scale (RPE) values were treated as continuous variables. Statistical significance was accepted at $\mathrm{p} \leq 0.05$. The effect size was evaluated with $\eta^{2}$ (Eta partial squared), where $0.01<\eta^{2}<0.06$ represents a small effect, $0.06<\eta^{2}<0.14$ a medium effect, and $\eta^{2}>0.14$ a large effect [3].

\section{RESULTS}

Because the subjects could not run faster than $16 \mathrm{~km} / \mathrm{h}$ on the NMCT, the $3 \times 4$ ANOVA with repeated measures was changed to a $3 \times 3$ model, where, in addition, the physiological and perceptual variables of the maximal $1000 \mathrm{~m}$ runs were compared with the measured variables in the NMCT at the different velocities. The running time during the maximal $1000 \mathrm{~m}$ run on the track and the motorized treadmill was on average $179 \pm 11 \mathrm{~s}$ $(2.59 \pm 11 \mathrm{~min})$, which equals a velocity of $20.1 \pm 1.0 \mathrm{~km} / \mathrm{h}$. A significant effect of increasing running velocity was found on oxygen uptake $(F=292.2$, $\mathrm{p}<0.001, \eta^{2}=0.97$; Figure 1), heart rate $\left(\mathrm{F}=141.3, \mathrm{p}<0.001, \eta^{2}=0.94\right.$; Figure 1), RPE ( $F=262.4, p<0.001, \eta^{2}=0.97$; Figure 2$)$, and blood lactate concentration $\left(\mathrm{F}=43.9, \mathrm{p}<0.001, \eta^{2}=0.94\right.$, Figure 2$)$. Post hoc comparison showed that with increasing velocity in all modalities, all of the physiological and perceptual variables increased, except for the blood lactate concentration on the motorized treadmill $(\mathrm{p}=0.059)$ and the track $(\mathrm{p}=0.900)$ from 12 to $14 \mathrm{~km} / \mathrm{h}$ (Figure 2). 


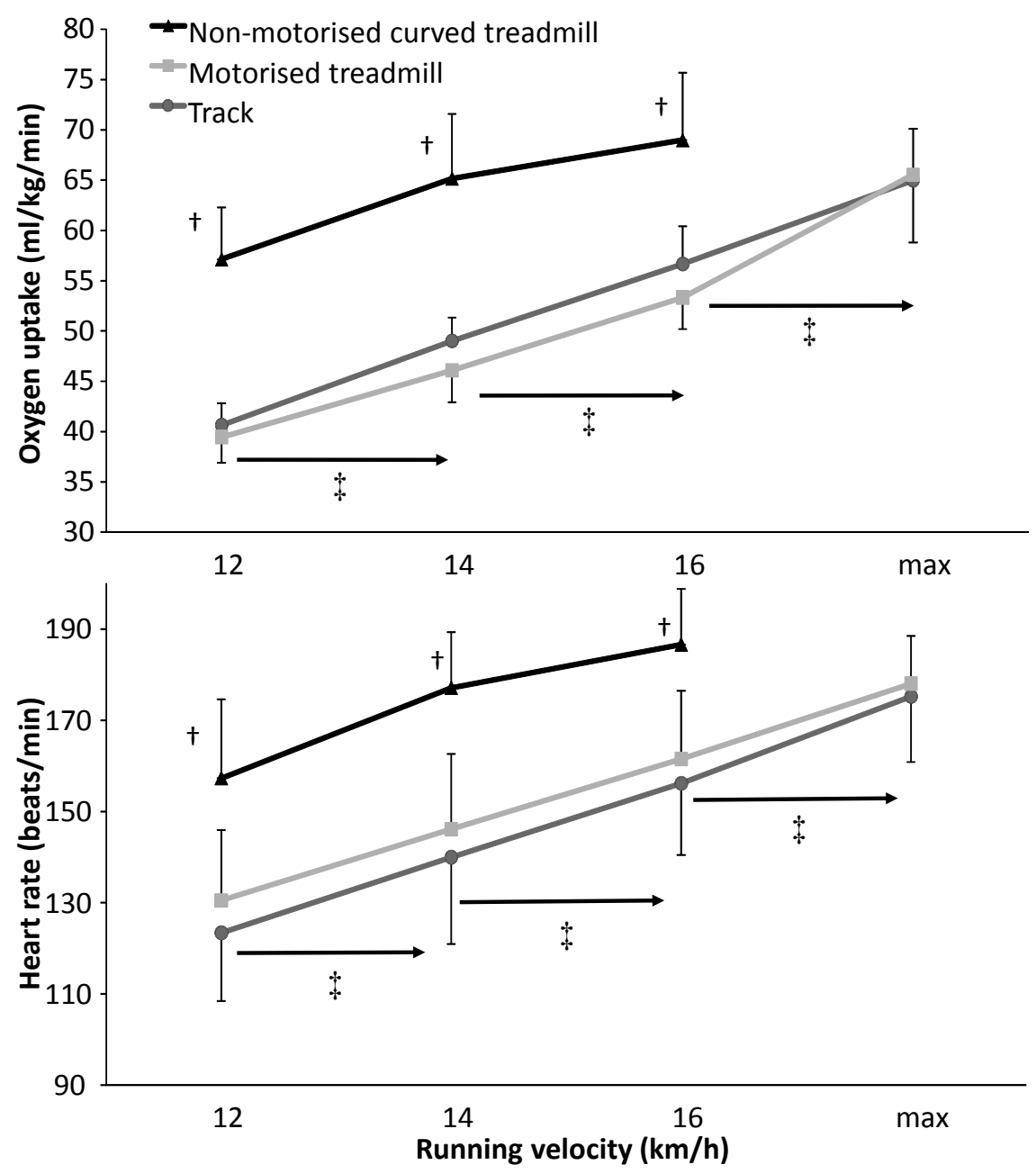

Figure 1. Maximal oxygen uptake and heart rate development (mean $\pm \mathrm{SD}$ ) at 12, 14, and $16 \mathrm{~km} / \mathrm{h}$ on the motorized treadmill, non-motorized curved treadmill, and indoor track. \#indicates a significant increase from one velocity to the next for all modalities on a $p<0.05$ level. 'indicates a significant difference $(p<0.05)$ with all other modalities at this velocity. 

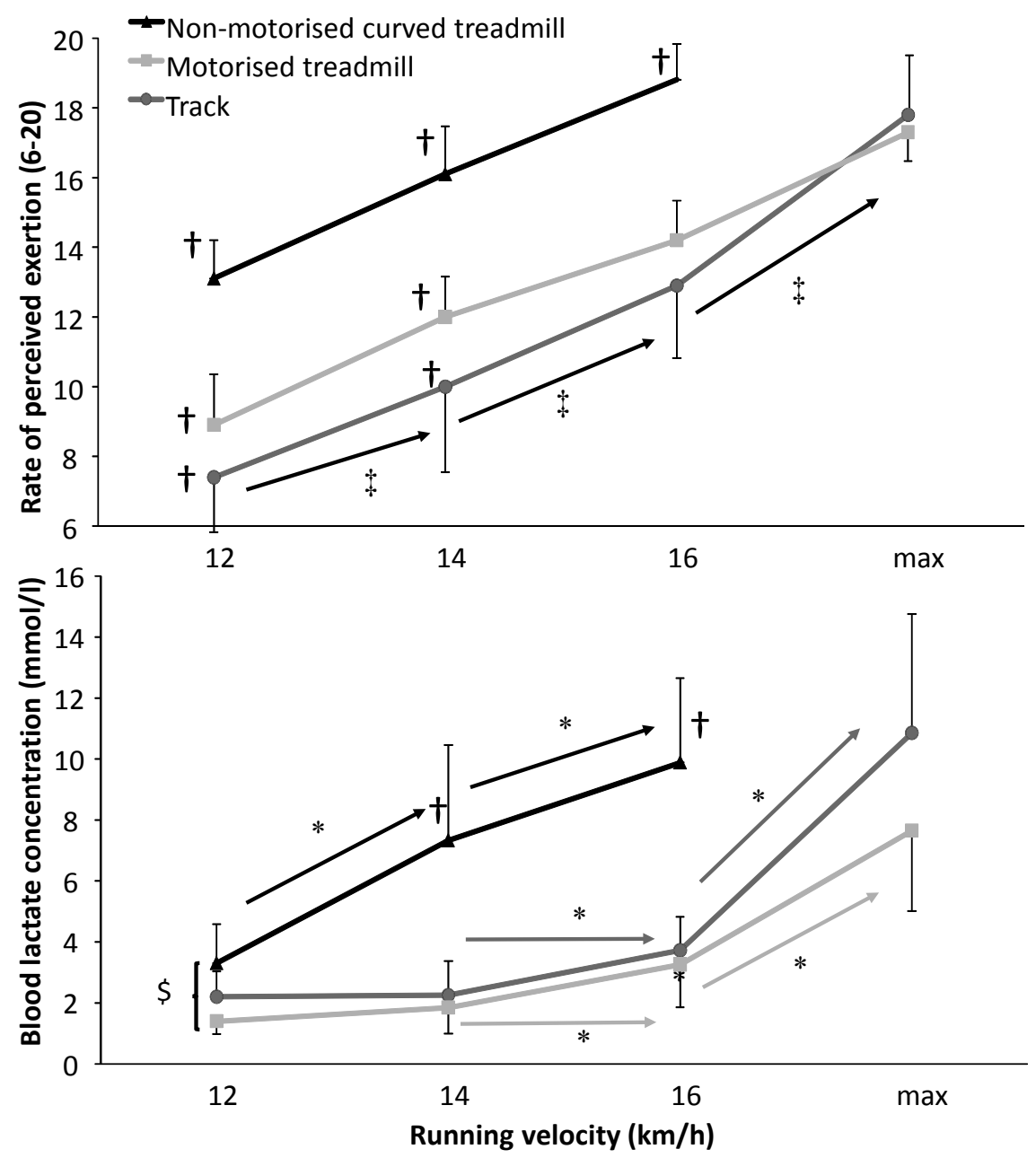

Figure 2. Rate of perceived exertion and blood lactate concentration (mean $\pm \mathrm{SD}$ ) at 12,14 , and $16 \mathrm{~km} / \mathrm{h}$ on the motorized treadmill, non-motorized curved treadmill, and indoor track. ‡indicates a significant increase from one velocity to the next for all modalities on a $p<0.05$ level. *indicates a significant increase from one velocity to the next for this modality on a $p<0.05$ level. ${ }^{+}$indicates a significant difference $p<0.05$ with all other modalities at this velocity. \$indicates a significant difference in blood lactate concentration between these two modalities at this velocity. 
A significant effect of modality was found on oxygen uptake ( $\mathrm{F}=56.8$, $\mathrm{p}<0.001, \eta^{2}=0.88$; Figure 1), heart rate $\left(\mathrm{F}=32.7, \mathrm{p}<0.001, \eta^{2}=0.78\right.$; Figure 1$)$, and RPE ( $F=52.1, \mathrm{p}<0.001, \eta^{2}=0.85$; Figure 2$)$, but not for blood lactate concentration $\left(\mathrm{F}=3.4, \mathrm{p}=0.10, \eta^{2}=0.53\right.$; Figure 2$)$. Post hoc comparison showed that on the NMCT at all velocities, all physiological and perceptual variables were higher than when running at a similar velocity on the other two modalities. Except for the blood lactate concentration, no significant difference was found between the NMCT and running on the track at $12 \mathrm{~km} / \mathrm{h}$ ( $\mathrm{p}=0.900$; Figure 2). In addition, a significantly higher RPE was found for running on a motorized treadmill compared with running on the track ( $\mathrm{p}=0.030$; Figure 2). No significant interaction effect was found for either the physiological or perceptual variables $\left(F \leq 3.13, p \geq 0.075, \eta^{2} \geq 0.28\right.$; Figures 1 and 2).

\section{DISCUSSION}

The main purpose of this study was to compare perceptual and physiological variables when running on an NMCT with running on an indoor track and a motorized treadmill. The main findings were, as hypothesized, that the physiological (oxygen uptake, heart rate, and blood lactate concentration) and perceptual (RPE) variables were higher when running on an NMCT than when running on the track or a motorized treadmill. No differences were found between running on a motorized treadmill and the track except for the RPE, which was lower when running on the track than on the motorized treadmill.

Oxygen uptake was on average $37 \%$ higher after the $1000 \mathrm{~m}$ runs, whereas the heart rate was on average $22 \%$ higher on the NMCT compared with the motorized treadmill and the track. These values were even higher than the producer of the NMCT advertises with: "burning up to $30 \%$ more calories than on any other treadmill". The findings were comparable with the study of Smoliga et al. [22] who also found that it costs more energy to walk and run on an NMCT compared to a motorized treadmill. However, they found even higher energy cost differences, from over $70 \%$ more oxygen uptake during walking and $43 \%$ during running at $8 \mathrm{~km} / \mathrm{h}$. The higher difference found between the two modalities can be explained by the fact that the authors did not perform a familiarization session before conducting the test [20]. Furthermore, the subjects in the study of Smoliga et al. [22] were a heterogeneous group of healthy recreational active and competitive males and females, which also may affect oxygen uptake results during the 
task. In addition, it seems that the difference in oxygen uptake between the NMCT and motorized treadmill decreases with increasing velocity [22]. In our study, a decrease in differences between the modalities was also found $(12 \mathrm{~km} / \mathrm{h}: 43 \% ; 14 \mathrm{~km} / \mathrm{h}: 37 \%$; and $16 \mathrm{~km} / \mathrm{h}: 25 \%)$. This decrease is explainable by the fact that at 14 and $16 \mathrm{~km} / \mathrm{h}$ a part of the energy necessary to run at these velocities on the NMCT probably came from anaerobic energy processes. When running at $12 \mathrm{~km} / \mathrm{h}$ on the NMCT, the subjects were already close to their anaerobic threshold, corresponding to the onset of blood lactate accumulation (OBLA) [15]. At 14 and $16 \mathrm{~km} / \mathrm{h}$ on the NMCT, subjects were over their anaerobic threshold, which was also shown by a flattening out of the oxygen uptake and heart rate at the increasing velocity. This caused lower differences in the $1000 \mathrm{~m}$ runs on the track and motorized treadmill, because on these modalities the main energy expenditure was still aerobic and under the anaerobic threshold, as shown by the blood lactate concentrations (see Figure 2).

The RPEs during the present study were also comparable to the findings of Smoliga et al. [22] and Stevens et al. [23]. Our subjects expressed the same RPE (motorized treadmill: 8.9; NMCT: 13 ) scores at the $12 \mathrm{~km} / \mathrm{h}$ velocity as the subjects in the study of Smoliga et al. [22] at their running velocity of $8 \mathrm{~km} / \mathrm{h}$, which also shows the difference in performance level between the two studies. In the 5-km runs in the study of Stevens et al. [23], the subjects reported an average RPE of 6 on the $0-10$ Borg scale [2], which is comparable to around 16 on the 6-20 Borg scale [1]. In the present study, these and higher RPEs were reported in the maximal $1000 \mathrm{~m}$ runs on the track $(20 \mathrm{~km} / \mathrm{h})$ and motorized treadmill $(20 \mathrm{~km} / \mathrm{h})$ and at 14 and $16 \mathrm{~km} / \mathrm{h}$ on the NMCT (Figure 2).

There was also a difference in RPE between the motorized treadmill and the track at 12 and $14 \mathrm{~km} / \mathrm{h}$, which can be explained by the fact that most trainings the subjects perform are on a track and not a treadmill. Furthermore, on the track you receive visual cues from moving surroundings, which makes it mentally easier to run compared with a surrounding that is not moving [12].

Our study showed that at every velocity the physiological and perceptual variables of NMCT running were higher compared with both track and motorized treadmill running. The findings of a higher heart rate and oxygen uptake in NMCT running compared to track and motorized treadmill running are consistent with other studies that conducted tests on larger, flat non-motorized treadmills in comparison with motorized treadmills $[5,9,21]$. De Witt et al. [5] stated that the higher metabolic costs on the non-motorized treadmill were mainly caused by the fact that the runner had 
to create belt motion, because the belt resistance causes extra force to propel. On the flat non-motorized treadmill, the subject has to lean forward while wearing a harness. This creates a moment from the center of pressure with the feet, which helps to propel the treadmill. To increase the velocity, more forward leaning is necessary. The producer of the NMCT states that the belt is nearly frictionless. However, the running belt also has a mass that has to be accelerated between every step to keep up the velocity, which causes high intrinsic resistance [10]. Besides the intrinsic resistance of the belt, the construction of the non-motorized treadmill (curved shape) in the present study causes another way to propel the belt motion. Due to the curved shape of the belt, leaning is not possible, because it would cause a rotation that could not be stopped by running faster. Therefore, in the present study, the subjects had to put their feet closer to the front of the curved belt to increase velocity. This allows a greater contribution of vertical force, which propels the belt [22].

Smoliga et al. [22] observed that subjects contacted an area of the treadmill that was approximately a 5 to 10 -degree gradient above the horizontal. This angle decreases throughout the stance phase. Thus, the incline of the curve of the treadmill may also be one of the main contributors of greater perceptual and physiological responses on the NMCT. This increases the force the subjects have to use to maintain a constant velocity. Lakomy [13] also found that more force is required to propel the treadmill belt with increasing velocity, which results in a faster increase in oxygen consumption and heart rate compared with running in graded exercise intensities on a motorized treadmill or a track (see Figure 1). While running on the track at a constant velocity, subjects only have to overcome wind resistance due to running velocity. Therefore, in the present study, running on a motorized treadmill was done at an incline of $1 \%$ to simulate wind resistance [12].

In a training situation, it is important that the training load is comparable when training on different running modalities. In the present study, it is shown that the running velocity on the NMCT has to be decreased by around $4 \mathrm{~km} / \mathrm{h}$ in order to have comparable physiological (oxygen uptake, heart rate and blood lactate concentration) and perceptual (RPE) responses with running on a track or motorized treadmill. The physiological (oxygen uptake, heart rate and blood lactate concentration) and perceptual (RPE) responses from $12 \mathrm{~km} / \mathrm{h}$ on the NMCT (see Figures 1 and 2) were similar to the responses at $16 \mathrm{~km} / \mathrm{h}$ on the track and motorized treadmill. Furthermore, the maximal running performance on the track, which was at $20 \mathrm{~km} / \mathrm{h}$, was comparable to the physiological responses at 14 and $16 \mathrm{~km} / \mathrm{h}$ on the NMCT. 
Therefore, it may be better to base training load upon heart rate intensity or RPE than on velocity when subjects and athletes utilize different locomotion modalities.

The subjects were all experienced in running with graded intensity on both a track and motorized treadmill, but they had only run one familiarization test on the NMCT. It has been suggested that a minimum of two familiarization trials, separated by at least 48 hours, should be required prior to experimental testing on an NMCT to improve reliability $[8,11]$. Lack of familiarity with the running modality has been shown to affect oxygen uptake [20]. However, great differences in both perceptual and physiological responses between NMCT running track and motorized treadmill running most likely would occur even if the subjects were fully familiarized with the modality. It might be that some effect could have been avoided with additional familiarization tests prior to the start of the test. Furthermore, the running surface was different between the modalities: the running surface on the track was Mondo, which is famous for being a fast track with little damping, which would have an influence on the energy expenditure during running [7]. The NMCT was from Woodway, which has a running surface with a lot of damping. Running on this surface would cost more energy than running on a hard, flat surface. The motorized treadmill surface was also different from the NMCT, which could influence the energy expenditure. Thus, in a future study the motorized treadmill should have the same running surface as the NMCT to avoid this influence.

Additionally, in this study, no electromyography of the involved muscles, stride rate measurements, or positional measurements were performed. This could have given more detailed information about the muscle behaviour and kinematics during running on the three running modalities. Thus, future studies should include these measurements.

Our study showed that running on an NMCT at three different velocities results in a higher oxygen uptake (37\%) and heart rate (22\%) and is subjectively much harder than running on a track or a motorized treadmill at the same velocities. The difference is around $4 \mathrm{~km} / \mathrm{h}$ when comparing the physiological and perceptual responses. Thus, when performing training sessions on an NMCT, subjects should subtract $4 \mathrm{~km} / \mathrm{h}$ from their regular pace on a track or motorized treadmill to get the same response. Furthermore, this type of non-motorized treadmill can be used as a type of strength training, such as hill running. However, further investigation should be initiated to determine what physiological adaptions the higher energy costs of NMCT 
running lead to in order to avoid altered running techniques caused by the use of an NMCT.

\section{ACKNOWLEDGMENTS}

This study was conducted without any funding from companies or manufacturers or outside organizations.

\section{REFERENCES}

1. Borg E, Kaijser L. (2006) A comparison between three rating scales for perceived exertion and two different work tests. Scand J Med Sci Sports, 16: 57-69

2. Borg GA. (1982) Psychophysical bases of perceived exertion. Med Sci Sports Exerc, 14: 377-381

3. Cohen J. (1988) Statistical power analysis for the behavioral sciences. Hillsdale, NJ, England: Lawrence Erlbaum Associates

4. Daniels JT. (1985) A physiologist's view of running economy. Med Sci Sports Exerc, 17: 332-338

5. De Witt JK, Lee SM, Wilson CA, Hagan RD. (2009) Determinants of time to fatigue during nonmotorized treadmill exercise. J Strength Cond Res, 23: 883890

6. Franks KA, Brown LE, Coburn JW, Kersey RD, Bottaro M. (2012) Effects of Motorized vs Non-Motorized Treadmill Training on Hamstring/Quadriceps Strength Ratios. J Sports Sci Med, 11: 71-76

7. Garcia-Perez JA, Perez-Soriano P, Llana S, Martinez-Nova A, Sanchez-Zuriaga D. (2013) Effect of overground vs treadmill running on plantar pressure: influence of fatigue. Gait Post, 38: 929-933

8. Gonzalez AM, Wells AJ, Hoffman JR, Stout JR, Fragala MS, Mangine GT, McCormack WP, Townsend JR, Jajtner AR, Emerson NS, Robinson Iv EH. (2013) Reliability of the Woodway curve (TM) non-motorized treadmill for assessing anaerobic performance. J Sports Sci Med, 12: 104-108

9. Hagan RD, De Witt JK, Laughin MS, Lee SMC, Loehr JA. (2010) Motorized and non-motorized treadmill evaluation: Physiologic responses and biomechanical aspects. Nat Aeron Space Admin Tech Spec Rep:

10. Highton JM, Lamb KL, Twist C, Nicholas C. (2012) The reliability and validity of short-distance sprint performance assessed on a nonmotorized treadmill. J Strength Cond Res, 26: 458-465

11. Hopker JG, Coleman DA, Wiles JD, Galbraith A. (2009) Familiarisation and reliability of sprint test indices during laboratory and field assessment. J Sports Sci Med, 8: 528-532 
12. Jones AM, Doust JH. (1996) A 1\% treadmill grade most accurately reflects the energetic cost of outdoor running. J Sports Sci, 14: 321-327

13. Lakomy HKA. (1987) The use of non-motorized treadmill for analysing sprint performance Ergonomics, 30: 627-637

14. Lee SMC, De Witt JK, Smith C, Laughlin MS, Loehr JA, Norcross J, Hagan RD. (2006) Physiologic responses and biomechanical aspects of motorized and nonmotorized treadmill exercise: A ground-based evaluation of treadmills for use on the International Space Station. Nat Aeron Space Admin Tech Spec Rep:

15. Mamen A, Laparidist C, van den Tillaar R. (2011) Precision in estimating maximal lactate steady state performance in running using a fixed blood lactate concentration or a delta value from an incremental lactate profile test. Int J Appl Sports Sci, 23: 212-224

16. Mangine GT, Hoffman JR, Gonzalez AM, Wells AJ, Townsend JR, Jajtner AR, McCormack WP, Robinson EH, Fragala MS, Fukuda DH, Stout JR. (2014) Speed, force, and power values produced from nonmotorized treadmill test are related to sprinting performance. J Strength Cond Res, 28: 1812-1819

17. Mc Naughton LR, Thompson D, Philips G, Backx K, Crickmore L. (2002) A comparison of the lactate Pro, Accusport, Analox GM7 and Kodak Ektachem lactate analysers in normal, hot and humid conditions. Int J Sports Med, 23: $130-135$

18. McCarron J, Hodgson TL, Smith MF. (2013) Brain drain: Evaluating the impact of increased cognitive load during self-paced running performance. Brit J Sports Med, 47: 13-17

19. Medbo JI, Mamen A, Holt Olsen O, Evertsen F. (2000) Examination of four different instruments for measuring blood lactate concentration. Scand J Clin Lab Invest, 60: 367-380

20. Morgan DW, Martin PE, Krahenbuhl GS. (1989) Factors affecting running economy. Sports Med, 7: 310-330

21. Otto RM, Wygand J, Flanagan K, Rowley E, McPhilliamy M, Stewart B. (1997) A comparison of metabolic response to walking on motorized and non-motorized treadmills. Med Sci Sports Exerc, 29: 203

22. Smoliga JM, Hegedus EJ, Ford KR. (2015) Increased physiologic intensity during walking and running on a non-motorized, curved treadmill. Phys Ther Sport, 16: 262-267

23. Stevens CJ, Hacene J, Wellham B, Sculley DV, Callister R, Taylor L, Dascombe BJ. (2015) The validity of endurance running performance on the Curve 3(TM) non-motorised treadmill. J Sports Sci, 33: 1141-1148

24. van Someren KA, Howatson G, Nunan D, Thatcher R, Shave R. (2005) Comparison of the Lactate Pro and Analox GM7 blood lactate analysers. Int J Sports Med, 26: 657-661

25. Williams KR. (1990) Relationships between distance running biomechanics and running economy. In: Cavanagh $\mathrm{PR}$, (ed). Biomechanics of distance running. Champaign, Ill.: Human Kinetics Publishers, 271-305 
Correspondence to:

Roland van den Tillaar PhD.

Department of Sports Science and Physical Education Nord University

Odins veg 23

7603 Levanger, Norway

Phone: +47-5767 1883

Fax: 0047-7411 2001

E-mail: roland.v.tillaar@nord.no 